\title{
Os resultados do implante coclear em crianças portadoras de Neuropatia Auditiva: revisão de literatura
}

\author{
The results of cochlear implants in children with Auditory Neuropathy: \\ literature review
}

\author{
Rafaela Carolina Lopez Silva ${ }^{1}$, Samantha Gomes Araújo ${ }^{2}$
}

\begin{abstract}
RESUMO
A Neuropatia Auditiva é um distúrbio na condução do estímulo sonoro, provavelmente relacionado à alteração da mielinização das fibras do nervo auditivo. Os avanços tecnológicos têm levado ao aumento nos recursos de diagnóstico e intervenção nos casos de deficiência auditiva por etiologia de Neuropatia Auditiva. O objetivo do presente estudo foi pesquisar na literatura os resultados obtidos em crianças com Neuropatia Auditiva após implante coclear. Foi realizado um trabalho de revisão bibliográfica em livros, periódicos e internet dos últimos dez anos sobre crianças com Neuropatia Auditiva usuárias de implante coclear. As pesquisas sugerem que a estimulação elétrica é capaz de compensar a dessincronia do nervo e que o implante coclear é um recurso para a (re)habilitação da audição em crianças portadoras de Neuropatia Auditiva. O uso do implante coclear permite melhoria significativa nas habilidades da audição e da comunicação. No entanto, as habilidades adquiridas parecem sofrer influência de diversos fatores como: tempo de uso do dispositivo, existência de um processo terapêutico sistemático, época do diagnóstico e a idade da criança.
\end{abstract}

DESCRITORES: Implante coclear; Audição; Criança; Deficiência auditiva/etiologia; Doenças do sistema nervoso periférico/ fisiopatologia

\section{INTRODUÇÃO}

Neuropatia Auditiva é o acometimento do nervo auditivo, que gera uma dessincronia na condução nervosa. Clinicamente, observa-se uma perda auditiva bilateral de grau variável e alteração na discriminação vocal. As Emissões Otoacústicas (EOA) apresentam-se normais e os Potenciais Evocados Auditivos de Tronco Encefálico (PEATE) não responsíveis ${ }^{(1)}$. Não está definida a localização precisa da alteração, podendo ser nas células ciliadas internas, nas sinapses entre as células ciliadas internas e o VIII par, no próprio VIII par (vestíbulo-coclear), ou ainda, em várias dessas estruturas ${ }^{(1-2)}$.

O diagnóstico da Neuropatia Auditiva é feito com base na aplicação de uma série de testes, em indivíduos com alteração específica da audição, ou em crianças pertencentes ao grupo de risco para deficiência auditiva, que consiste em complicações na gestação, no parto ou no período neonatal ${ }^{(3-4)}$.

(1) Mestre, Docente do Curso de Fonoaudiologia da Pontifícia Universidade Católica de Minas Gerais - PUC-Minas - Belo Horizonte (MG), Brasil.

(2) Fonoaudióloga clínica, graduada pela Pontifícia Universidade Católica de Minas Gerais - PUC-Minas - Belo Horizonte (MG), Brasil.

Endereço para correspondência: Rafaela Carolina Lopez Silva. Rua Dom Arisitides Porto, 252/301, Bairro Coração Eucarístico, Belo Horizonte - MG,

CEP 30535-450. E-mail: rafaelalopezaudio@yahoo.com.br

Recebido em: 28/8/2006; Aceito em: 15/1/2007
A Neuropatia Auditiva é descrita em pacientes de todas as idades, com audição compatível com a função coclear normal e alteração na função neural ${ }^{(5)}$.

Para as crianças candidatas a cirurgia de implante coclear é importante à realização da bateria completa de exames (EOA, PEATE, avaliação comportamental e imitanciometria), possibilitando um diagnóstico mais preciso e melhores condições de êxito na terapêutica ${ }^{(2,6)}$.

O implante coclear (IC) substitui parcialmente as funções da cóclea, transformando a energia sonora em sinais elétricos ${ }^{(1-2)}$. No entanto, antes da realização da cirurgia, é necessário determinar se o paciente é candidato ao implante e, para esta decisão, exige-se uma equipe multiprofissional (médicos, enfermeiros, psicólogos, fonoaudiólogos, assistentes sociais) $)^{(7)}$.

Indivíduos portadores de perda auditiva severa e/ou profunda e com grande dificuldade na discriminação vocal, que se beneficiam pouco com o uso dos aparelhos de amplificação sonora, pois dificilmente se obtém uma melhora significativa e simétrica dos limiares auditivos, principalmente em frequiências altas, são candidatos ao implante $\operatorname{coclear}^{\left({ }^{8}\right)}$.

O implante coclear oferece a possibilidade do limiar auditivo bastante equivalente em todas as freqüências, uma vez que este é alcançado, aumentando-se ou diminuindo-se, artificialmente, a intensidade da corrente elétrica em cada eletrodo $^{(8)}$. 
O quadro clínico da Neuropatia Auditiva está muitas vezes relacionado à grande dificuldade ou dificuldade desproporcional em compreensão da fala ${ }^{(3)}$. Assim, a reabilitação em pacientes portadores de Neuropatia Auditiva é um desafio, pois o nervo auditivo contribui para uma percepção da fala severamente prejudicada, comprometendo, desta forma, a codificação temporal da informação. A amplificação sonora não contribui na deficiência da codificação da informação, embora os pacientes portadores de Neuropatia Auditiva possam demonstrar melhoras nos limiares auditivos por meio do uso de aparelhos de amplificação sonora. Outra questão é a heterogeneidade dos portadores de Neuropatia Auditiva em relação aos achados audiológicos e neurológicos, além da etiologia incerta ${ }^{(9)}$.

Torna-se necessária uma análise voltada para os resultados obtidos com o uso do implante coclear para verificar a efetividade do mesmo em crianças portadoras de Neuropatia Auditiva.

Com a possibilidade do implante coclear oferecer benefícios para a aquisição da linguagem em crianças deficientes auditivas, desenvolvimento auditivo e melhoria na sincronia neural, este estudo tem como objetivo pesquisar os resultados obtidos em crianças com Neuropatia Auditiva usuárias de IC.

Esta pesquisa é relevante, uma vez que o uso do implante coclear em pacientes com deficiência auditiva por etiologia de Neuropatia Auditiva é um recurso terapêutico recentemente disponível e ainda restrito, exigindo assim maiores investigações sobre este tema por parte dos profissionais envolvidos.

Foi realizado um trabalho de revisão bibliográfica sobre as crianças com Neuropatia Auditiva usuárias de implante coclear. Foram selecionados materiais de sites, como: Pubmed, Bireme, Medline, periódicos nacionais e internacionais, monografias, teses e anais, do período de 1995 a 2005, sobre Neuropatia Auditiva, implante coclear e crianças portadoras de Neuropatia Auditiva, que se submeteram à cirurgia de implante coclear e seus resultados.

\section{REVISÃO DA LITERATURA}

\section{Neuropatia Auditiva}

É bastante ampla a investigação acerca da neuropatia, tanto que vários autores têm se dedicado a esse estudo ${ }^{(3)}$. Alterações auditivas podem preceder em muitos anos o aparecimento da neuropatia periférica ${ }^{(6)}$.

A Neuropatia Auditiva é definida como uma perda da sincronia na condução nervosa, provavelmente relacionada à alteração da mielinização das fibras do nervo auditivo ${ }^{(1)}$. A transmissão do estímulo falha em produzir despolarização uniforme, suficiente para que se obtenham os potenciais auditivamente evocados de tronco encefálico ${ }^{(3)}$.

Clinicamente, observa-se uma perda auditiva bilateral, simétrica ou não, com grau variando entre leve e profundo; a curva audiométrica pode registrar um traçado plano, ascendente ou descendente; discriminação vocal incompatível com o grau da perda, ou seja, a reposta da logoaudiometria é mais baixa que o esperado pelo grau da perda auditiva. Pode haver alteração dos reflexos acústicos estapedianos. As Emissões Otoacústicas (EOA) apresentam-se normais, porém não há ausência do efeito supressor nas vias auditivas eferentes. Durante a realização do Potencial Evocado Auditivo de Tronco Encefálico (PEATE) há ausência ou alteração de respostas, estando presente o microfonismo coclear $^{(3-4)}$.

A época de início da Neuropatia Auditiva é dividida em dois grupos: antes e após a aquisição da fala. A aquisição da fala pela criança com Neuropatia Auditiva é muito difícil, pelo transtorno auditivo e por ser uma faixa etária na qual são comuns as etiologias vinculadas às complicações de gestação, parto e período neonatal, como, por exemplo, a hiperbilirrubinemia, a prematuridade e heranças genéticas ${ }^{(3)}$.

A precisão dos novos equipamentos e o grande avanço tecnológico levaram a um aumento nos recursos de diagnóstico de perdas auditivas na infância. Entre os exames audiológicos para crianças temos: audiometria de observação comportamental, audiometria com reforço visual, observação das respostas a estímulos verbais, audiometria lúdica ou convencional, imitância acústica, EOA e PEATE ${ }^{(2,10)}$.

O PEATE é um método importante na avaliação do sistema auditivo central, identificando possíveis alterações na sincronia neural. É um exame que avalia a integridade da via auditiva no tronco encefálico ${ }^{(2,10)}$.

\section{Deficiência auditiva e implante coclear}

Os primeiros anos de vida são importantes para um bom desenvolvimento auditivo e da linguagem. Assim, priorizase o diagnóstico precoce da deficiência auditiva, pois durante este período ocorre a maturação do sistema auditivo. Essa maturação se dá em duas fases: na primeira ocorre a do sistema auditivo periférico, por volta da $20^{\mathrm{a}}$ semana de gestação, e na segunda ocorre a das vias auditivas ao longo do sistema nervoso central (SNC), por volta dos 18 meses de idade. Portanto, a imaturidade do SNC não pode levar a considerações precisas a respeito das respostas evocadas auditi$\operatorname{vas}^{(2)}$.

O distúrbio auditivo na infância pode provocar efeitos diversos na comunicação e uma série de deficiências secundárias, como: alterações perceptuais, de fala, de comunicação, cognitivas, emocionais, sociais, educacionais e intelectuais $^{(9)}$. Os aparelhos de amplificação sonora podem ser um recurso no tratamento da perda auditiva de diversos graus, porém pacientes com discriminação menor que $40 \%$ em teste de reconhecimento de sentenças em apresentação aberta, são candidatos a uma segunda alternativa de reabilitação: o implante coclear (IC) ${ }^{(8)}$.

O IC destina-se a pacientes portadores de perda auditiva neurossensorial bilateral de grau severo e/ou profundo, que não se beneficiam do aparelho de amplificação sonora individual (AASI) $)^{(7,11)}$.

O IC estimula o VIII par craniano por meio de eletrodos, levando a informação sonora, previamente transformada em sinais elétricos, diretamente ao nervo auditivo ${ }^{(7)}$.

$\mathrm{O}$ IC é formado por componentes externos e internos. Os componentes externos são: microfone, processador de fala e 
antena transmissora. O componente interno é um receptorestimulador, que inclui a antena interna, colocada cirurgicamente junto ao osso do crânio, atrás da orelha, sob a pele, e o feixe de eletrodos, posicionado dentro da cóclea ${ }^{(12)}$.

$\mathrm{O}$ sistema de IC funciona em frações de segundos. Os sons ambientais são captados por meio do microfone direcional retroauricular e enviados, por meio de um cabo, para o processador de fala, que filtra, analisa, digitaliza e converte os sons de entrada em sinais codificados. Estes são enviados, por meio de um cabo, do processador de fala para a antena transmissora, que remete esses sinais transcutaneamente para o receptor (estimulador por rádio freqüência). O receptor converte os códigos em sinais eletrônicos e os envia para o feixe de eletrodos inseridos na cóclea. Por sua vez, os eletrodos estimulam as fibras nervosas e os impulsos neurais resultantes seguem pelo sistema nervoso central até os centros auditivos do cérebro, local onde os sinais serão interpretados ${ }^{(12)}$.

Antes da realização da cirurgia é necessário determinar se o paciente é candidato ao $\mathrm{IC}^{(9)}$. A idade mínima permitida para a cirurgia é de 12 meses $^{(13)}$. Outros estudos demonstram que a idade mínima para a implantação é de 18 meses, tempo mínimo para a identificação e confirmação do diagnóstico de perda auditiva ${ }^{(13)}$.

Crianças menores de três anos que foram implantadas, após quatro a cinco anos de uso do IC, obtêm melhor desempenho na produção da fala e na sua inteligibilidade. Isso de deve à plasticidade da via auditiva. A plasticidade é a habilidade das conexões neuronais em serem modificadas pelo crescimento das sinapses, produzindo estimulações adequadas. O processo de plasticidade começa a se limitar rapidamente, a partir dos seis anos de vida; assim, essa idade é considerada como um período crítico para a implantação e para o desenvolvimento da via auditiva pós-implante ${ }^{(13)}$.

Determinar se o paciente é candidato ao IC é uma tarefa que exige uma equipe multiprofissional, considerando o indivíduo em todos os aspectos: médicos, audiológicos, psicoemocionais, sociais e culturais ${ }^{(5)}$. A equipe básica é composta por: médicos, fonoaudiólogos, enfermeiras, psicólogos, assistentes sociais e outros técnicos, que acompanham os pacientes em todas as etapas (avaliação, retornos pré-cirúrgicos, hospitalização e retornos pós-cirúgicos para acompanhamento $)^{(14)}$.

Devem ainda ser considerados os critérios eletrofisiológicos e médico-cirúrgicos: perda auditiva neurossensorial bilateral de grau severo e/ou profundo, com nenhum benefício com os AASI; portadores de deficiência mental ou problemas psiquiátricos não são candidatos ao implante; recursos para a (re)habilitação na cidade de origem; motivação da família e do paciente; o nervo auditivo e o sistema auditivo central devem estar íntegros (exames de tomografia de alta resolução e a ressonância magnética identificam anormalidades que possam comprometer a cirurgia do implante $)^{(15)}$.

A orelha a ser implantada deverá ser aquela com menores resíduos auditivos, na tentativa de preservar o lado melhor para eventual amplificação ${ }^{(11)}$.

A corrente necessária para eliciar a sensação auditiva é diferente para cada indivíduo; assim, os parâmetros de estimulação elétrica são ajustados individualmente, adequando-se às necessidades dos usuários. Esse processo é denominado de mapeamento. Por meio do software, o fonoaudiólogo acessa a interface e envia sinais em níveis precisos, para o processador de fala e, conseqüentemente, para o paciente ${ }^{(12)}$.

Atualmente, há equipamentos que permitem um registro intra-operatório do funcionamento dos eletrodos e do potencial de ação do nervo. Esta medida denomina-se Telemetria de Respostas Neurais, ou seja, medição de uma informação de um lugar a outro. Há um software incluído em equipamentos de implante coclear que funciona como uma miniunidade de potencial evocado ${ }^{(13)}$. A Telemetria permite confirmar a integridade do dispositivo interno, na determinação de quais eletrodos podem ser incluídos em um determinado mapa, as melhores velocidades de estimulação e estratégias de codificação da fala ${ }^{(12)}$.

\section{Neuropatia Auditiva e implante coclear}

A Neuropatia Auditiva é uma situação de contra-indicação à cirurgia de implante coclear, porém sabe-se que esta doença é operável, e os resultados obtidos são vários, podendo ser equivalentes aos de pacientes que não têm esse diagnóstico ${ }^{(13)}$.

É importante o acompanhamento audiológico de crianças com Neuropatia Auditiva, já que podem adquirir uma perda coclear com o passar do tempo. Nestes casos, estas crianças tornam-se candidatas ao $\mathrm{IC}^{(4)}$.

Em um estudo de caso de uma criança portadora de Neuropatia Auditiva que recebeu um IC, houve melhora significativa na percepção da fala, nos achados pós-implante. $\mathrm{O}$ estudo recomenda a implantação de forma mais precoce nos pacientes portadores de Neuropatia Auditiva e ainda sugere que a excitação elétrica nestes casos é uma opção viável ${ }^{(9)}$.

Em outra pesquisa com cinco crianças com diagnóstico de Neuropatia Auditiva, implantadas com o dispositivo Nucleus 24, depois da colocação dos dispositivos internos do implante coclear, no intra-operatório, foram feitas medidas dos eletrodos. No pré-operatório, as crianças foram submetidas a exames audiológicos, pediátrico, neurológico, genéticos, radiológicos e médicos. As avaliações da audição destas crianças incluíram técnicas de audiometria comportamental, medidas objetivas da função da orelha média e interna, PEATE e, em alguns casos, eletococleografia transtimpânica (EcoG). Cada criança teve acompanhamento regular, durante um ano, para avaliar as condições médicas gerais e as habilidades da audição e comunicação. As cinco crianças implantadas não tiveram complicações médicas e do dispositivo do IC no pós-operatório. Todas as crianças mostraram bons resultados na Telemetria de Resposta Neural e todos, menos um caso que não foi testado, mostraram evidência de respostas no PEATE e melhorias significativas nas habilidades de percepção de fala e habilidades de comunicação após o uso do $\mathrm{IC}^{(16)}$.

Outros pesquisadores estudaram e compararam os resultados obtidos nos testes de percepção de fala, reflexo acústico eletricamente evocado e PEATE em quatro crianças com Neuropatia Auditiva que receberam IC com um grupo con- 
trole de crianças implantadas com deficiência auditiva neurossensorial severa a profunda da mesma faixa etária, levando em consideração a idade da implantação e o uso do IC. De acordo com os autores, os resultados sugerem que não foram observadas diferenças significativas entre os resultados do grupo de Neuropatia Auditiva e do grupo controle. Os resultados das crianças com Neuropatia nos exames e nos testes (PEATE, Reflexo Acústico Eletricamente Evocado e percepção da fala) foram compatíveis com os dados obtidos pelo grupo controle. Foram obtidas respostas do PEATE em pelos menos dois dos três eletrodos testados, e Reflexo Acústico Eletricamente Evocado presente sem decadência. Assim, os autores concluíram que há possibilidade do IC melhorar a sincronia neural e contribuir para a percepção de fala das crianças com Neuropatia Auditiva ${ }^{(17)}$.

Em um estudo realizado no Center for Hearing and Deafness Research, em Cincinnati, entre os anos de 1993 e 2001, foram testados 18 pacientes com diagnóstico de Neuropatia Auditiva que se submeteram à cirurgia de implante coclear, sendo 11 do gênero feminino e sete do gênero masculino, com três pares de irmãos, incluindo um par de gêmeos idênticos. Doze dos 18 pacientes apresentavam fatores de risco clássicos para Neuropatia Auditiva. O grau da perda auditiva variou de severo a profundo. Todas as crianças implantadas mostraram melhoria no desenvolvimento da audição e percepção da fala, mas esta melhoria era variável entre os indivíduos. Este estudo sugeriu o sucesso de implantação nestes pacientes e que crianças com Neuropatia Auditiva apresentam uma lesão no sistema auditivo que pode ser compensada por meio da estimulação elétrica ${ }^{(18)}$.

Mais recentemente, pesquisadores acompanharam a evolução audiológica em cinco crianças portadoras de Neuropatia Auditiva, no pré e pós-cirúrgico do IC. Todos os pacientes se submeteram às medidas audiológicas (Audiometria e PEATE) e teste de percepção da fala. Os resultados demonstraram que os pacientes tiveram benefícios com o IC. Os autores concluíram que o IC pode ser um recurso para a habilitação da audição em crianças portadoras de Neuropatia Auditiva ${ }^{(19)}$.

Foi realizado um estudo de caso em uma criança diagnosticada como portadora de Neuropatia Auditiva e que recebeu IC. O dispositivo implantado foi o Nucleus 24. No pré-cirúrgico, incluíram avaliação médica, audiológica (PEATE e Emissões Otoacústicas Transientes) e estudo de imagem. Depois do procedimento cirúrgico foram realizados teste de percepção da fala, exames audiológicos, mapeamento e Telemetria de resposta neural. Nenhuma discriminação para a linguagem foi observada antes da realização da cirurgia de IC. Melhorias significativas das habilidades da audição e da percepção da fala foram observadas após dez meses da cirurgia de IC. Houve melhora na intenção comunicativa e na capacidade para elaborar orações de três palavras. Este estudo concluiu que o estímulo elétrico do IC foi um instrumento importante para a reabilitação e melhoria das habilidades auditivas e de fala do paciente portador de Neuropatia Auditiva ${ }^{(20)}$

Pesquisadores, com o objetivo de determinar as habilidades de fala em crianças portadoras de Neuropatia Auditiva que se submeteram à cirurgia de $\mathrm{IC}$, realizaram um estudo com oito crianças de 15 meses a dez anos de idade. Foram realizados testes para habilidades de fala expressiva, receptiva e testes audiológicos. Os resultados deste estudo sugerem que as crianças com Neuropatia Auditiva que usam implante coclear desenvolvem a fala de forma semelhante às crianças que possuem perda auditiva e que se submeteram à cirurgia de IC. Os pesquisadores concluíram que o IC é um instrumento de reabilitação em crianças com Neuropatia Auditiva $^{(21)}$.

Um estudo comparou dez crianças portadoras de Neuropatia Auditiva, com dez crianças portadoras de perda auditiva por outras etiologias. Todas as 20 crianças receberam IC. Foram usadas várias medidas para comparar os resultados entre os dois grupos, como: audiometria, limiar de desconforto, teste de percepção da fala, Telemetria, reflexo estapediano acústico, nível de escolaridade e modo de comunicação. Os resultados deste estudo demonstraram que não houve diferença significativa do benefício do IC entre os dois grupos. O estudo sugeriu o uso do IC como uma opção viável para crianças portadoras de Neuropatia Auditiva(22).

Os mesmos autores em um outro estudo investigaram o desempenho do IC em duas crianças (uma com aplasia do nervo auditivo coclear bilateral e outra, portadora de Neuropatia Auditiva). Também foram estudados três adultos com ossificação coclear. As duas crianças que não tinham nenhuma habilidade de audição, com dois meses de ativação do IC puderam reconhecer sons e palavras, mostrando melhoria progressiva no desempenho da audição ${ }^{(23)}$.

Uma pesquisa ainda em andamento, da Faculdade de Odontologia de Bauru, teve o objetivo de relatar os resultados preliminares da performance de produção de fala de crianças com Neuropatia Auditiva pré-lingual usuárias de IC. Foram avaliadas três crianças (idade variando de cinco anos e três meses a seis anos e três meses), usuárias de IC, há pelo menos cinco meses, por meio de repetição de palavras, nomeação de figuras e discurso espontâneo. Uma criança apresentou vocalizações não articuladas em todas as tarefas. Duas crianças apresentaram produções inteligíveis, sendo que o fonema $/ \mathrm{p} /$ foi produzido corretamente de modo mais freqüente. A melhor performance foi obtida pela criança com maior tempo de uso do IC e processo terapêutico mais consistente. Uma das conclusões parciais deste estudo mostrou que a produção de fala de crianças com Neuropatia Auditiva usuárias de IC parece sofrer influência do tempo de uso do dispositivo e da existência de um processo terapêutico sistemático $^{(24)}$.

\section{DISCUSSÃO}

É recente a descrição de Neuropatia Auditiva e observase um número restrito de publicações relacionadas aos resultados do IC em crianças portadoras de Neuropatia Auditiva, principalmente na literatura nacional.

A Neuropatia Auditiva é um distúrbio na condução do estímulo sonoro, provavelmente relacionada à alteração da mielinização das fibras do nervo auditivo ${ }^{(1)}$. Estudos afirmam que para o diagnóstico desta desordem, são combinadas análises dos resultados de avaliações atuais, em indivíduos com 
alteração específica da audição, ou em crianças pertencentes ao grupo de risco para deficiência auditiva ${ }^{(3-4)}$.

Estudos concordam que o avanço tecnológico leva ao aumento nos recursos de diagnóstico de perdas auditivas ${ }^{(2,10)}$. Conseqüentemente, também há o aumento do diagnóstico da Neuropatia Auditiva.

As pesquisas caracterizam a Neuropatia Auditiva como: resposta anormal, presença de microfonismo coclear no teste de PEATE, alteração na discriminação vocal, e EAO presentes, o que sugere o funcionamento adequado das células ciliadas externas ${ }^{(3-4)}$. A localização precisa da Neuropatia Auditiva não está definida, podendo ser desde a célula ciliada interna até o VIII par (vestíbulo-coclear) ou em várias dessas estruturas $^{(1-2)}$. Os achados audiológicos em pacientes portadores de Neuropatia Auditiva são heterogêneos e ela possui uma etiologia incerta.

As pesquisas concordam que o IC é indicado para pacientes portadores de perda auditiva neurossensorial bilateral de grau severo e/ou profundo, que não apresentam benefícios com o uso do aparelho de amplificação sonora individual ${ }^{(6,11)}$.

Não há uma concordância entre os autores no que diz respeito à idade para a implantação. A idade mínima para a cirurgia de IC é de 12 meses $^{(12)}$. Outros estudos sugerem a idade mínima para a implantação de 18 meses, sendo o tempo mínimo para a identificação e confirmação do diagnóstico de perda auditiva ${ }^{(14)}$. Ainda, outro estudo recomenda a implantação coclear de forma mais precoce nos portadores de Neuropatia Auditiva $^{(9)}$. No entanto, outro estudo sugere que devido à plasticidade auditiva, crianças menores de três anos que são implantadas, após quatro a cinco anos de uso do IC, obtém melhores performances na produção da fala e na sua inteligibilidade $^{(13)}$. Além disso, prioriza-se o diagnóstico da deficiência auditiva nos primeiros anos de vida, pois durante este período ocorre a maturação do sistema auditivo, importante para um bom desenvolvimento auditivo e de linguagem ${ }^{(2)}$.

O uso dos aparelhos de amplificação sonora em indivíduos portadores de perda auditiva severa e/ou profunda e com grande dificuldade na discriminação vocal oferece pouco benefício, pois dificilmente se obtém uma melhora simétrica dos limiares auditivos, principalmente nas freqüências altas. O IC diferencia-se do AASI por estimular diretamente o nervo auditivo por meio de sinais elétricos ${ }^{(5)}$, oferecer a possibilidade do limiar auditivo bastante equivalente em todas as freqüências ${ }^{(8)}$ e pela possibilidade de os parâmetros de estimulação elétrica serem ajustados individualmente, adequando-se às necessidades dos usuários (mapeamento). São enviados sinais em níveis precisos para o processador de fala e, conseqüentemente, para o paciente ${ }^{(12)}$. Desta forma, o IC atualmente é um recurso para pacientes portadores de Neuropatia Auditiva.

Autores sugerem o acompanhamento audiológico de crianças portadoras de Neuropatia Auditiva, pela possibilidade da amplificação sonora individual ocasionar perda auditiva neurossensorial com o passar do tempo ${ }^{(4)}$.

Portadores de Neuropatia Auditiva possuem a percepção da fala severamente prejudicada, pois o distúrbio na condução do estímulo auditivo ocasiona uma codificação temporal anormal ${ }^{(9)}$. Desta forma, é um desafio à reabilitação das crianças portadoras de neuropatia, devido ao prejuízo na condução do estímulo até o Sistema Auditivo Central, comprometendo o desenvolvimento da maturação auditiva e do processamento auditivo, importantes para o desenvolvimento da linguagem oral.

Os autores estudados concordam que ocorre melhora significativa na percepção da fala, nas habilidades de audição e comunicação nas crianças usuárias de implante coclear ${ }^{(9,16-}$ 18,20-21). No entanto, os estudos sugerem que os benefícios do uso do IC, em crianças portadoras de Neuropatia Auditiva, com relação à aquisição da linguagem e desenvolvimento das habilidades auditivas, estejam relacionados ao tempo de uso deste dispositivo, existência do processo de reabilitação fonoaudiológica, época do diagnóstico e idade da criança.

As pesquisas concordam que a excitação elétrica é capaz de compensar a dessincronia do nervo auditivo ${ }^{(9,16-18,20)}$ e que o IC é um recurso para a (re)habilitação da audição em crianças portadoras de Neuropatia Auditiva(19-21).

Pesquisas sugerem melhoras no teste PEATE, pesquisa do reflexo eletricamente evocado acusticamente e na Telemetria de Resposta Neural, após a implantação ${ }^{(16-17)}$.

Estudos concordam também que a criança com Neuropatia Auditiva usuárias de IC possuem benefícios e desenvolvem a fala de forma semelhante às crianças portadoras de perda auditiva e que se submeteram à cirurgia de $\mathrm{IC}^{(21-22)}$.

Não há concordância entre os estudos com relação à época em que ocorrem as melhorias nas habilidades da audição e da linguagem, após a cirurgia de IC. No estudo do caso de uma criança diagnosticada como portadora de Neuropatia Auditiva usuária de IC, nenhuma discriminação para a linguagem foi observada antes da realização da cirurgia, ocorrendo melhoria significativa das habilidades da audição e da percepção da fala, após dez meses da cirurgia de $\mathrm{IC}^{(20)}$. Autores estudaram o desempenho do IC em uma criança portadora de Neuropatia Auditiva, que não tinha nenhuma habilidade de audição, podendo reconhecer sons e palavras após dois meses de ativação do implante, mostrando melhoria progressiva no desempenho da audição $^{(23)}$.

\section{COMENTÁRIOS FINAIS}

Os primeiros anos de vida são importantes para o desenvolvimento da linguagem e da maturação do sistema auditivo. Assim, o distúrbio auditivo na infância pode provocar um grande impacto negativo, na vida da criança com relação à comunicação, psicológico, social e aprendizagem.

O avanço tecnológico leva ao aumento do diagnóstico de desordens auditivas na infância.

O IC atualmente é um recurso disponível para pacientes com perda auditiva neurossensorial bilateral de grau severo e/ou profundo, pois estimula o VIII par craniano, levando a informação sonora diretamente ao nervo auditivo. Além disso, o IC permite que a estimulação elétrica seja ajustada individualmente, adequando-se às necessidades dos usuários.

Não há um consenso com relação à idade mínima permitida para a cirurgia. No entanto, recomenda-se a implantação de forma mais precoce, devido à plasticidade da via auditiva. 
A excitação elétrica é capaz de compensar a dessincronia do nervo auditivo. Assim, o IC é um recurso para a (re)habilitação da audição em crianças portadoras de neuropatia auditiva.

Crianças com Neuropatia Auditiva, usuárias de implante coclear, recebem benefícios de forma semelhante às crianças portadoras de perda auditiva, que se submeteram à cirurgia de IC.

A produção de fala e as habilidades adquiridas por crianças com Neuropatia Auditiva, usuárias de IC parece sofrer influência de diversos fatores como: tempo de uso do dispo- sitivo, existência de um processo terapêutico sistemático, existência do processo de reabilitação fonoaudiológica, época do diagnóstico e idade da criança.

O bom desempenho da audição é um ganho progressivo. Ocorre melhoria significativa nas habilidades da audição e da percepção da fala, na intenção comunicativa, na capacidade para elaborar orações, no reconhecimento dos sons.

Ressaltamos que se torna necessária a realização de outros estudos que permitam a análise qualitativa dos benefícios do implante coclear em crianças, principalmente portadoras de Neuropatia Auditiva.

\begin{abstract}
Auditory Neuropathy is a disorder in the transmission of the sound stimulus, probably related to mielinization alterations of auditory nerve fibers. Technology advances have been increasing diagnostic and intervention resources in cases of hearing loss caused by auditory neuropathy. The aim of the present study was to research the available literature about the results obtained with children with Auditory Neuropathy after cochlear implants. An ample review was conducted on books, articles and the internet, over the last 10 years, regarding children with Auditory Neuropathy, users of cochlear implants. The available data suggest that electric stimulation can compensate neural asynchrony and that cochlear implants aid in the rehabilitation process of children with Auditory Neuropathy. The use of cochlear implant allows significant improvement in communication and hearing abilities. However, the acquired abilities seem to suffer influence from many factors, such as: length of time using the device, the existence of a systematic therapeutic process, when the diagnosis was carried out, and the child's age.
\end{abstract}

KEYWORDS: Cochlear implantation; Hearing; Children; Hearing loss/etiology;Peripheral nervous system diseases/physiopathology

\title{
REFERÊNCIAS
}

1. Doyle KJ, Sininger Y, Starr A. Auditory neuropathy in childhood. Laryngoscope. 1998;108(9):1374-7.

2. Ogeda ECM, Matas CG. Neuropatia auditiva por Kernicterus: estudo de caso. Pró-Fono. 2002;14(2):247-52.

3. Berlin CI. Auditory neuropathy. Using OEAs and ABR from screening to management. Semin Hear. 1999;20(4):307-15.

4. Spinelli M, Fávero-Breuel ML, Silva CMS. Neuropatia auditiva: aspectos clínicos, diagnósticos e terapêuticos. Rev Bras Otorrinolaringol. 2001;67(6):863-7.

5. Hood LJ. Auditory neuropathy: what is it and what can we do about it? Hear J. 1998;51(8):10-8.

6. Starr A, Picton TW, Sininger Y, Hood LJ, Berlin CI. Auditory neuropathy. Brain. 1996;119(Pt 3):741-53.

7. Parra VM, Soares TCB, Iorio MCM, Chiari BM. Implante coclear: protocolo/síntese pré-operatório e caracterização da população encaminhada. Pró-Fono. 2001;13(1):30-6.

8. Bento RF, Brito Neto R, Castilho AM, Gómez VG, Giorgi SB, Guedes MC. Resultados auditivos com o implante coclear multicanal em pacientes submetidos a cirurgia no Hospital das Clínicas da Faculdade de Medicina da Universidade de São Paulo. Rev Bras Otorrinolaringol. 2004;70(5):632-7.

9. Trautwein PG, Sininger YS, Nelson R. Cochlear implantation of auditory neuropathy. J Am Acad Audiol. 2000;11(6):309-15.

10. Parra VM, Matas CG. Estudo das características audiológicas em casos de neuropatia auditiva. Pró-Fono. 2002;14(2): 241-6.

11. Gomez MVSG, Guedes MC, Ornelas CG, Sant'Anna SBG, Brito Neto RV. Preservação da audição residual em pacientes usuários de implante coclear multicanal: estudo piloto. Rev Bras Otorrinolaringol. 2002;68(5):698-702.

12. Rizzi FML, Bevilacqua MC. Efeitos do número e localização dos eletrodos na cóclea na percepção da fala de indivíduos pós-linguais implantados. Rev Bras Otorrinolaringol. 2003;69(3):364-9.

13. Diamante VG, Pallares N. Estado actual y futuro de los implantes cocleares. Rev Otorrinolaringol Cir Cabeza Cuello. 2003;63(3):197-206.

14. Mesquita ST, Canoas JW, Costa Filho OA. Implante coclear: quem são seus usuários. Rev Soc Bras Fonoaudiol. 2002;3(13):267-273.

15. Moraes TV, Zeigelboim BS, Bevilacqua MC, Jacob LCB. Indicação de implante coclear: tendências atuais. Acta AWHO. 2001;20(4):229-37.

16. Morant Ventura A, Orts Alborch M, García Callejo J, Pitarch Ribas MI, Marco Algarra J. [Auditory neuropathies in infants]. Acta Otorrinolaringol Esp. 2000;51(6):530-4. Spanish.

17. Buss E, Labadie RF, Brown CJ, Gross AJ, Grose JH, Pillsbury HC. Outcome of cochlear implantation in pediatric auditory neuropathy. Otol Neurotol. 2002;23(3):328-32.

18. Madden C, Hilbert L, Rutter M, Greinwald J, Choo D. Pediatric cochlear implantation in auditory neuropathy. Otol Neurotol. 2002;23(2):163-8.

19. Lefrancois R, Moran L, Schramm D, Pickard L. Cochlear implantation in children with auditory neuropathy. In: 9th Symposium Abstract Book; 2003 April; Washington (DC); 2003. p. 219.

20. Bevilacqua MC, Costa OA, Martinho AC. Auditory neuropathy and cochlear implants: case study. In: 9th Symposium Abstract Book; 2003 April; Washington (DC); 2003. p.144.

21. Somarriba K, et al. Auditory neuropathy: language skills and hearing results with cochlear implantation. In: 9th Symposium Abstract Book; 2003 April; Washington (DC); 2003. p. 223.

22. Colletti V, Carner M, Miorelli V, Guida M, Colletti L, Fiorino F. Cochlear implant failure: is an auditory brainstem implant the answer? Acta Otolaryngol. 2004;124(4):353-7.

23. Colletti V, Fiorino FG, Carner M, Miorelli V, Guida M, Colletti L. Auditory brainstem implant as a salvage treatment after unsuccessful cochlear implantation. Otol Neurotol. 2004;25(4):485-96; discussion 496.

24. Rodrigues LCB, Ferrari DV, Costa AD, Spinardi ACP. Produção de fala de crianças com neuropatia auditiva usuárias de implante coclear resultados preliminares. [cited 2005 Jun 8]. Disponível em: http:// www.usp.br/siicusp/12osiicusp/ficha2096.htm. 